\title{
A Biomimetic MEMS Direction Finding Acoustic Sensor based on MetalMUMPs Process
}

\author{
Syed Osama Bin Islam a, Muhammad Altaf Hussain ${ }^{\mathrm{b}}$, Muhammad Shoaib ${ }^{\mathrm{c}}$ and M. Haris Amir ${ }^{\mathrm{d}}$
}

\begin{abstract}
This paper presents the design and finite element method (FEM) modeling of MEMS acoustic sensor optimized using the design rules of the commercially available Metal-Multi User MEMS Process (MetalMUMPs). The acoustic sensor is modeled with a structural layer of Nickel with $20 \mu \mathrm{m}$ thickness. The acoustic sensor is designed for the detection of sound direction where the displacement amplitude of the sensor plates vary with the applied pressure and angle at specific sound frequency. Moreover, the stress induced in the sensor, corresponding to the input sound waves, is analyzed using FEM simulations and geometric modifications are made to have the stress in the sensor microstructure less than the yield stress of the Nickel. This allows to minimize the effect of mechanical fatigue on the proposed MEMS acoustic sensor performance.
\end{abstract}

Keywords-Acoustic Sensor, MEMS, PTM, MetalMUMPs, FEM, Yield Strength.

\section{Introduction}

Micro Electro Mechanical System (MEMS) sound sensors offer a promising technology platform for a wide range of applications due to their size, low power consumption and high sensitivity. Various applications for MEMs acoustic sensors include torque and tyre pressure sensors, gas sensors, biosensors for medical applications, industrial and commercial applications [1]. An important aspect of sound sensors is their use in military application like detection of sound source of large and small weapons as highlighted in [2]. A confined explosive charge is used in conventional firearms for propulsion of bullet from the weapon's barrel. The acoustic energy and the shock wave due to explosion are collectively called the muzzle blast. This blast lasts for a maximum of 3 milliseconds [3]. The muzzle blast acts like a primary acoustic signal if recorded from a microphone located close to the weapon. This muzzle blast ranges over a span of frequencies as it involves sound energies from various sources that include the sound of blast, bullet hissing and shock wave. The wide frequency ranges can be sampled through special microphones in combination with high-speed, data acquisition computer systems. This resolves the impulses into discrete acoustic signals that can be related to different sources. The recorded shots can be digitized and processed by computer using a Fast Fourier Transform procedure to synthesize comparable octave band results.

Syed Osama Bin Islam (Author)

Muhammad Altaf Hussain (Author)

Muhammad Shoaib (Author)

M. Haris Amir (Author)

National University of Sciences and Technology

Pakistan
In [4] acoustical characterization of five guns was carried out, to determine their suitability as impulse sources. The 0.38 caliber was found most suitable and the peak frequency was found to be around $900 \mathrm{~Hz}$. In [5] it was revealed that for large-calibers the main part of the acoustic spectral energy lies below $400 \mathrm{~Hz}$ and peak $(16-100 \mathrm{~Hz})$, whereas for small-calibers (rifles) it lies between $150-2,500 \mathrm{~Hz}$ and peak (900-1,500 Hz). In [6] analysis of the FFT of a $9 \mathrm{~mm}$ gun was carried out. One can observe low magnitudes at low frequencies and higher magnitudes at higher frequencies. The peak frequencies were found between $800 \mathrm{~Hz}$ to 1000 Hz.

Directional sound sensing microphones, imitating the hearing system of the Ormia Ochracea fly are becoming popular research area recently. The operation of these directional sound sensors requires understanding of interaction of the MEMS structure with the incident sound field. Ormia Ochracea is a fly which is commonly found in Mexico and southern parts of United States. It is a parasite and studies revealed that on the island of Kauai in Hawaii, it has destroyed huge population of crickets [7]. The fly is of small size almost $500 \mu \mathrm{m}$, yellow colour and has the quality to identify the direction from where the cricket is chirping based on incident sound waves. The reproduction of the fly thus depends on locating living crickets [8]. The crickets usually produce the chirping sound to attract females at dawn or dusk thus increasing dependency on hearing system [9]. The fly hearing system consists of two eardrums which are called prosternal tympanic membranes (PTMs) that receive the sound waves incident on them. The PTMs transfer the energy to auditory sensory organs which are in pair called bulbae acusticae. A ridge-like ingrowth of the exoskeleton connects the bulbae acusticae to the membranes called (apodeme), which looks like a stiff rod. The mechanical connection among two PTMs is the key element of the fly's hearing system that is also called the intertympanal bridge. PTM close to the sound source is called (ipsilateral) and PTM away from the sound source is called (contralateral). When the incident sound wave hits the PTM near to the sound source, the PTM away moves in the direction opposite via intertympanal bridge. This would produce a motion just like children seesaw. The contralateral, which has already moved due to the mechanical connection between the PTMs, will now resist the force due to the incident sound wave in contact acting in opposite direction. As a result, a bending motion is produced across the intertympanal bridge. The effect of this is that the ipsilateral will be having larger amplitude than the contralateral. Therefore, the neural sensing system of the ear near to the sound source will have more effect of sound energy than the one far from the source, however response will be symmetric if incident sound is normal to PTM [10]. 


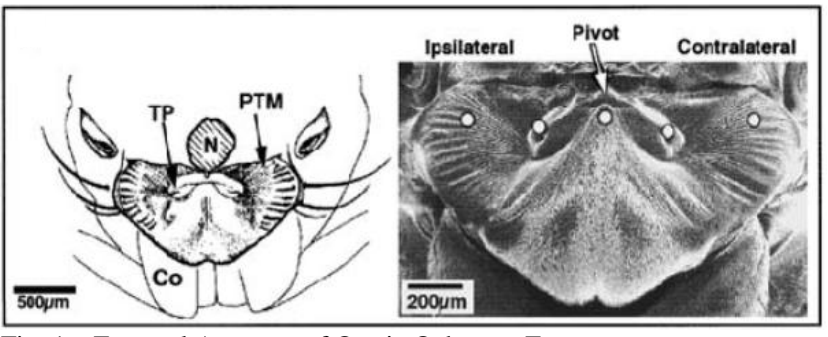

Fig. 1. External Anatomy of Ormia Ochracea Ears

Similar type of phenomenon can be seen in pressure gradient microphones. A pressure gradient microphone is the one in which the two sides of the diaphragm are exposed to the incident sound. A pressure differential (gradient) is therefore produced between the two sides of the diaphragm. However no pressure differential occurs when the incident sound wave is parallel to the plane of the diaphragm. A silicon-micromachined prototype was presented in [11]. Multiple piezoelectric sensing ports were used simultaneously for transduction of the sensing structure.

Miles et al. [10] found that the PTMs of the fly's hearing system are mechanically coupled and overall structure has two natural resonant frequencies. The membranes move out of phase with each other at the first mode in a pure rocking fashion due to the minute pressure difference amongst them. The membranes move in phase at second mode, which results in a pure bending motion about the tympanal bridge. The MEMS sensor behaves just like a pressure gradient microphone when the sound wave interacts with its both sides. Miles showed that the amplitude of sensor's vibration has cosine dependence with direction of sound. The amplitude of plates at first and second mode is therefore dependent on cosine angle of incident sound which can be either in-phase or out-of-phase. In [12] theoretical investigation of the fly ears was carried out to estimate the time delay between the two ears when sound is incident at an angle. A 2 Degree of freedom mathematical model was used to estimate the time responses of two ears when subjected to typical shockwave produced by gunshot. Directional sensitivity of the fly ears was calculated that came out to be $1.3 \mu \mathrm{s} / \mathrm{deg}$ and compared with conventional microphone whose sensitivity is $0.06 \mu \mathrm{s} / \mathrm{deg}$. The time delay will cause the structure to produce a rocking motion when two ears are coupled and would result in low deflection. In [13] it was revealed that coupling increases the time and amplitude difference of Ormia Ochracea ears. A mathematical model was used to calculate the difference of arrival using CRB technique. It was found that in the coupled model the errors are reduced when estimating direction of arrival. It was also shown that a larger SNR is required to achieve better results. In [14] a sensor was designed using SOIMUMPs process and was optimized for directional sensitivity. It was revealed that the sensitivity of the directional cues is dependent on the strength of coupling (intertympanal bridge). If the coupling is strong the directional sensitivity is high, whereas the directional sensitivity is low when coupling is weak. A dual optimization point was found between the two extremes. However the sensitivity would still be low when compared with sensor operating at bending frequency and 0 degree incident angle due to cosine dependence and inter-aural- time-difference. In [15] a sensor was designed using SOIMUMPs process with interdigitated comb fingers for transduction. The incorporation of transducers still confirms the cosine dependence on incident sound. It was also referred that to ascertain the direction of sound, amplitude of pressure is required which can be measured through omni directional microphone. In [16] a device was designed based on PiezoMUMPs process. Simulations of the modal, displacement and stress analysis were carried out. The sensitivity of the device was found to be $3.8 \mathrm{nV} / \mathrm{Pa}$ calculated from piezo material.

In [17] Daniel et al. designed a sensor that operates at its bending resonance frequency and had verified the cosine angle dependence. This means that determination of the direction became ambiguous when symmetric reading is obtained about the normal axis. To remove the ambiguity it was proposed to place a pair of sensor at canted angle and direction is to be determined from cumulated readings. The key idea presented in the paper was based on the operation of Ormia Ochracea type acoustic sensors primarily at their bending resonant frequency. This needs insight of harmonic analysis of the structure which has interacted with an incident sound wave of particular frequency. The resonant frequency which was proposed to be the operating frequency is dependent on mass of the two wings and the dimensions of the bridge. The sensor was designed with a commercial process of Silicon-on-Insulator Multi User Manufacturing Process (SOIMUMPs) by MEMSCAP.

The metal-multi user MEMS process (MetalMUMPs) proposed for fabrication in this work is a commercially available low cost method. Electroplated nickel is the primary material used in the process. There are certain design rules that define the limit of the process [18].

The objectives of this research are:

- To design a MEMS acoustic sensor prototype considering the design rules of MetalMUMPs process.

- To optimize the design specifically to find direction of fire from small caliber guns with sound waves of particular frequency.

- To analyze the stresses induced in the microstructure corresponding to the input sound waves.

\section{Design of Proposed MEMS Acoustic Sensor}

A micro-sensor is designed following the design rules [18] stated by MetalMUMPs process in Solidworks software. The prototype is specifically designed for small calibre weapons based applications for required frequency range $(800-1000 \mathrm{~Hz})$. Subsequently it was imported to ANSYS for its structural analysis. Modes of resonant frequencies of the structure are calculated by performing modal analysis. Subsequently harmonic and stress analysis 
are performed, wherein inputs of pressure and incident angle of acoustic waves were given. Considering no damping validation of the results of adapted design from [17] is carried out and compared with ones obtained from the MetalMUMPs based prototype.

\section{A. Prototype Design}

Fig. 2 shows the design of the proposed MEMS acoustic sensor. The plates are clamped by a bridge, which in turn clamped in the center by another small bridge. Hence the structure contains four beams which act when force is applied (two under torsion and two under bending). The plates will be deflected by the incident sound pressure at particular angle falling on them.

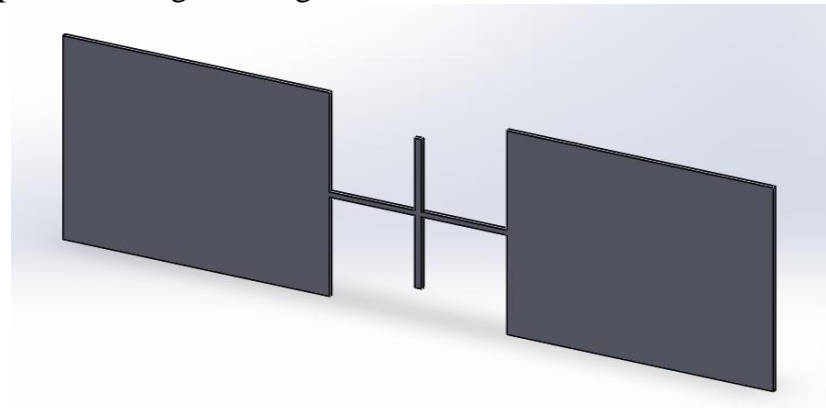

Fig. 2. Prototype designed in solid works

A pair of plates are incorporated each having a length and width of $1.2 \mathrm{~mm}$. Two beams attached with each plate are designed having a total length of $385 \mu \mathrm{m}$, width of $30 \mu \mathrm{m}$ and thickness of $20 \mu \mathrm{m}$. Fixed-free beam configuration can be seen. The central two beams are of length $425 \mu \mathrm{m}$, width $30 \mu \mathrm{m}$ and thickness of $20 \mu \mathrm{m}$. Minimum feature size is considered $5 \mu \mathrm{m}$ as stated in latest specifications of MetalMUMPs design rules. Young's modulus of nickel is kept $159 \times 10^{3} \mathrm{MPa}$ [19], Poison ratio 0.22 and density is kept $8.9 \times 10^{-15} \mathrm{~kg} / \mu \mathrm{m}^{3}\left(8900 \mathrm{~kg} / \mathrm{m}^{3}\right)$.

\section{B. Mathematical Formulation}

The displacement in beam is related to the force acting at some point and the spring constant of the beam. The bending stiffness $K$ of a flexible pivot with a constant rectangular section without tensile load is expressed as:

$$
K=\frac{C E I}{L^{a}}
$$

where $I$ is moment of inertia,

$$
I=\frac{w t^{a}}{12}
$$

The resonant frequency of the structure can be found from the equation.

$$
w=\sqrt{\frac{K}{m}}
$$

where,

$w=$ Width of the flexible pivot

$t=$ Thickness of the beam

$E=$ Young's modulus of nickel

$I=$ Moment of inertia

$L=$ Length of beam

$C$ is a constant determined by the end to end configuration $m=$ Mass of the wings

\section{FEM Analysis of the MEMS Acoustic Sensor}

Modal analysis of the structure was carried out followed by structural analysis. Details are covered as under:

\section{A. Modal Analysis}

Modal analysis of the structure is shown in Table I. Deformation at first mode of resonant frequency shown in Fig. 3. is called rocking mode, whereas deformation at second mode of frequency shown in Fig. 4. is called bending mode.

TABLE I

MODAL ANALYSIS IN ANSYS

\begin{tabular}{ccc}
\hline \hline Serial & Mode & Frequency \\
\hline 1 & 1 & 636.33 \\
2 & 2 & 984.24 \\
3 & 3 & 1213.3 \\
4 & 4 & 1420.3 \\
5 & 5 & 2266.4 \\
6 & 6 & 2515.2 \\
\hline \hline
\end{tabular}



Fig. 3. $1^{\text {st }}$ mode Frequency response (Rocking mode $-636.33 \mathrm{~Hz}$ )

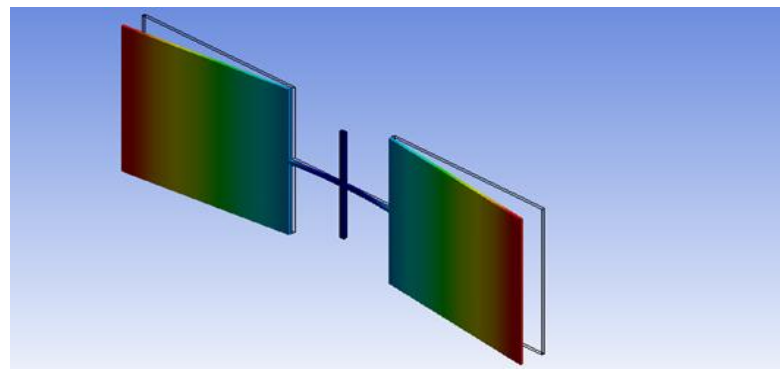

Fig. 4. $2^{\text {nd }}$ mode Frequency response (Bending mode $-984.24 \mathrm{~Hz}$ )

\section{B. Structural Analysis}

The response of a sensor is calculated by varying sound pressure as shown in Fig. 5. To draw maximum output incident sound angle is considered normal to the sensor wings $\left(\theta=0^{\circ}\right)$. The data in the figure shows that the sound pressure has linear relationship with response. The slope of the line gives sensitivity of about $21.6 \mu \mathrm{m} / \mathrm{Pa}$. It is to mention that pressure is applied without the bending resonant frequency. 
Proc. of the Sixth International Conference on Advances in Mechanical and Robotics Engineering - AMRE 2017. Copyright (C) Institute of Research Engineers and Doctors. All rights reserved.

ISBN: 978-1-63248-140-5 doi: 10.15224/ 978-1-63248-140-5-36

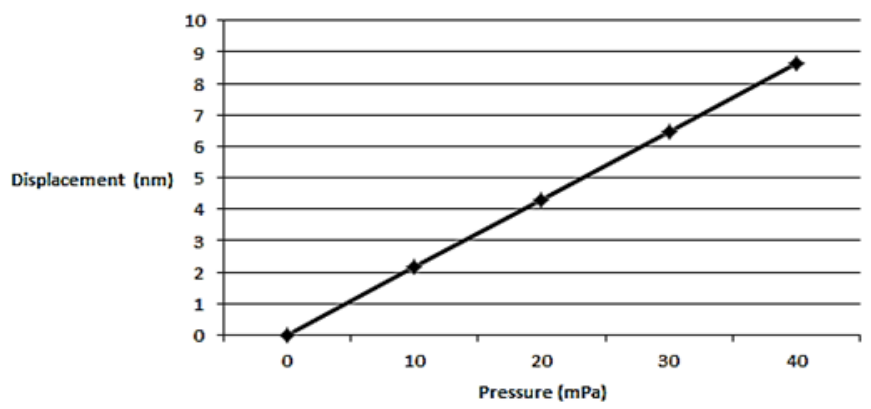

Fig. 5. Displacement against applied Pressure without Resonant Frequency

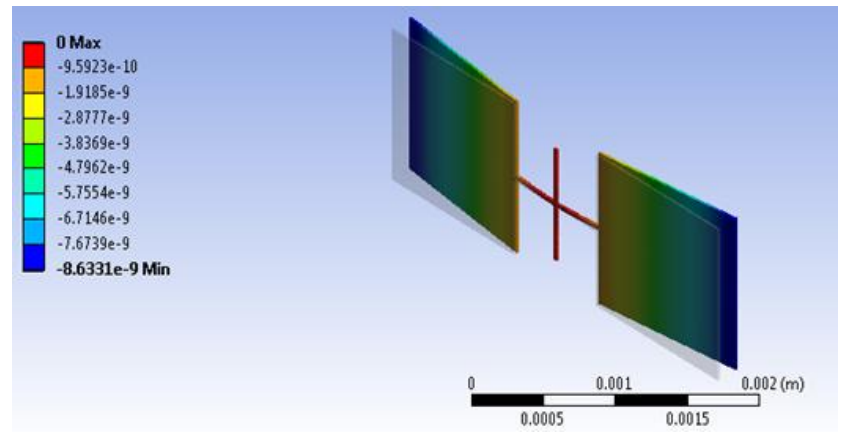

Fig.6. Sensor Response corresponding to Pressure without Resonant Frequency

\section{Harmonic Analysis}

The measured bending resonant frequency of the sensor is about $984.24 \mathrm{~Hz}$, which in actual is dependent on the mass of the wings and dimensions of the bridge in the middle. Again the response of a sensor is calculated by varying sound pressure using sound incident normal to the sensor wings $\left(\theta=0^{\circ}\right)$ for the maximum output, as shown in Fig. 7. The sound frequency was set to $984.24 \mathrm{~Hz}$ during the measurement. The data in the figure again shows that the sound pressure has linear relationship with the response. The slope of the line gives sensitivity of about $43.54 \mathrm{~mm} / \mathrm{Pa}$.

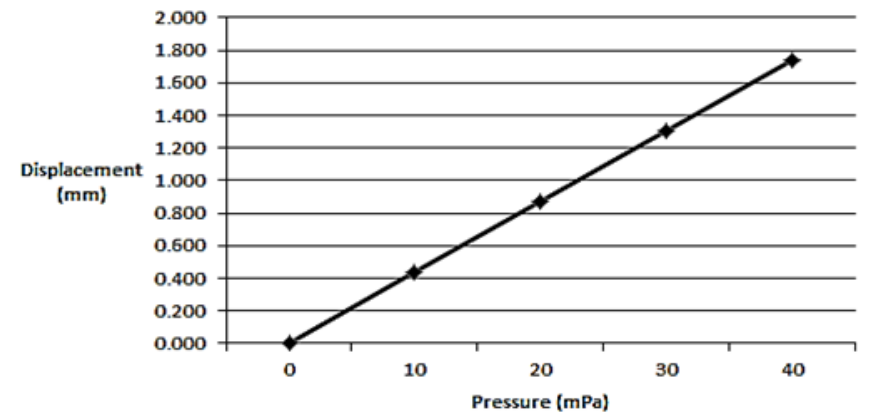

Fig. 7. Displacement against Pressure with Bending Resonant Frequency



Fig.8.Sensor Response corresponding to Pressure with Resonant Frequency

\section{Response against Incident Angle}

The angle of incident sound was varied for $40 \mathrm{mPa}$ and an angle ambiguity is seen mirrored about incident angle 0 . This angle ambiguity is proposed to be removed by placing two sensors at canted angle as was suggested in [17].

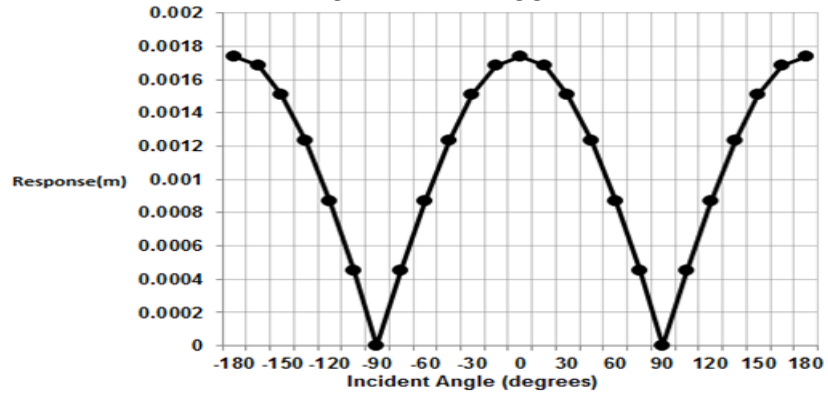

Fig. 9. Displacement with respect to change in Incident Angle

\section{E. Stress Analysis}

The stress analysis at the bending frequency revealed that the stresses higher than yield stress (160 MPa) are achieved when structure is subjected to pressure around $1.1 \mathrm{mPa}$, which is undesirable. This declares the pressure limit for the designed structure. To reduce the stress concentration seen in Fig. 10 a modification in design is proposed.

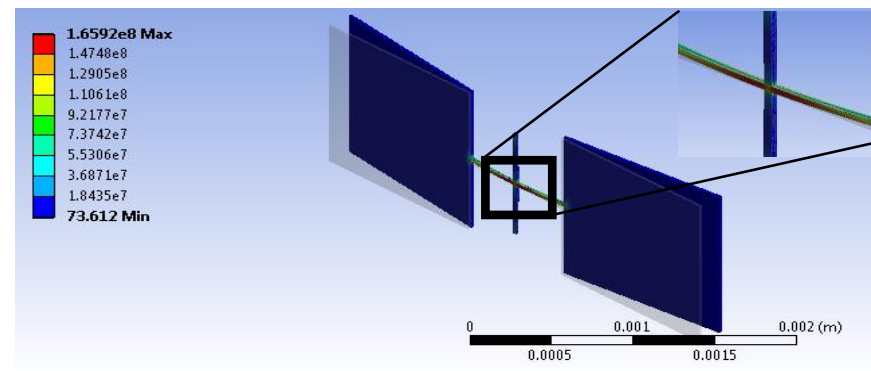

Fig. 10. Stress Analysis for Harmonic Response

\section{F. Modification in Structure}

The stress concentration at the edges of the beam can be reduced by chamfering the edges; however this will change the resonant frequency of the structure. By introducing the chamfer as shown in Fig. 11, the maximum stresses at $1.1 \mathrm{mPa}$ pressure are reduced below yield stress. To bring the resonant frequency within the required range, the dimensions of the beam as stated in equation (1) and (2) can be modified. Thickness is fixed by the MetalMUMPs process; hence width and length can only be modified. In this study length of the beams are modified to $500 \mu \mathrm{m}$. To further reduce the stress limit or to increase the pressure range, extent of chamfer can be increased.

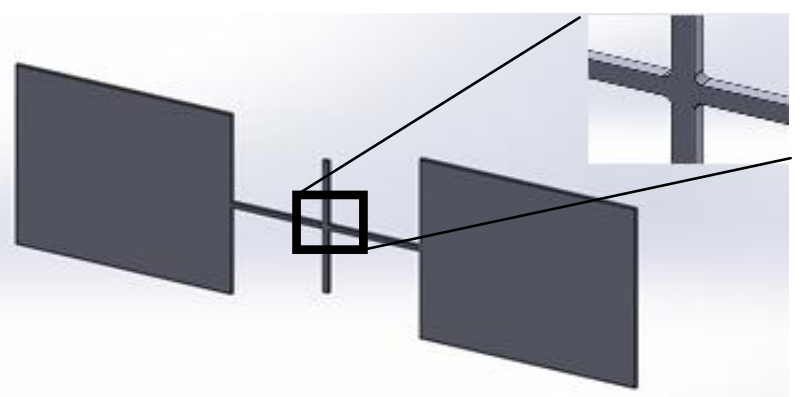

Fig. 11. Modified Design with Chamfered Edges 


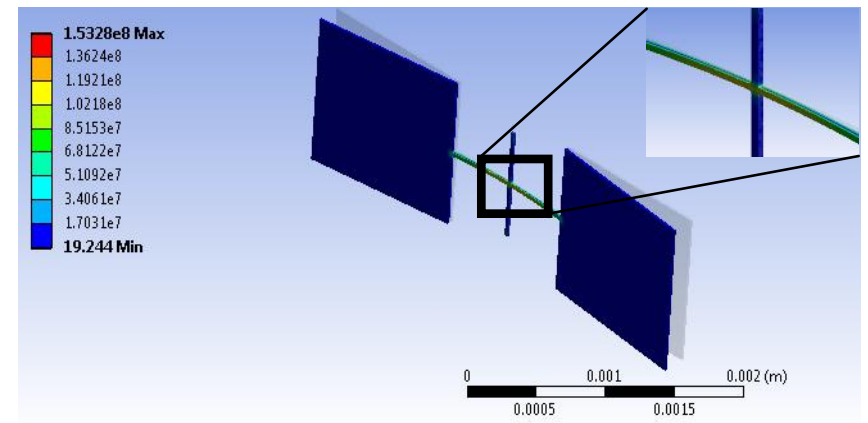

Fig. 12. Stress Concentrations after Modification in Structure

\section{G. Comparison}

A Comparison of designed structure with the design presented in [17] made from SOIMUMPs process is carried out in which design specifications, resonant frequencies and sensitivity are considered. The comparison is presented in Table II in which effects after modification are also included. It can be seen that the sensitivity of the proposed sensor has improved when modeled using design specifications of the MetalMUMPs process. However when the structure is modified to reduce the stress, the sensitivity of the sensor depreciated minutely but still in $\mathrm{mm} / \mathrm{Pa}$ range i.e. $31.53 \mathrm{~mm} / \mathrm{Pa}$. An optimization is to be carried out while introducing extent of chamfer, as it increases the stiffness of the beams at the centre, thus reducing the sensitivity. The length of the bridge therefore is increased in the modified design to bring the resonant frequencies in required range.

TABLE II

COMPARISON OF DESIGNEd STRUCTURE WITH SOIMUMPS DESIGN

\begin{tabular}{|l|c|c|c|}
\hline & $\begin{array}{c}\text { SOIMUMPs } \\
\text { Design }\end{array}$ & $\begin{array}{c}\text { MetalMUMPs } \\
\text { Design }\end{array}$ & $\begin{array}{c}\text { Modified } \\
\text { Design }\end{array}$ \\
\hline Bridge length $(\mu \mathrm{m})$ & 800 & 800 & 1050 \\
\hline Bridge width $(\mu \mathrm{m})$ & 30 & 30 & 30 \\
\hline Bridge thickness $(\mu \mathrm{m})$ & 25 & 20 & 20 \\
\hline Plate length $(\mathrm{mm})$ & 1.2 & 1.2 & 1.2 \\
\hline Plate width $(\mathrm{mm})$ & 1.2 & 1.2 & 1.2 \\
\hline Plate thickness $(\mu \mathrm{m})$ & 25 & 20 & 20 \\
\hline Support length $(\mu \mathrm{m})$ & 880 & 880 & 880 \\
\hline Support width $(\mu \mathrm{m})$ & 30 & 30 & 30 \\
\hline Support thickness $(\mu \mathrm{m})$ & 20 & 20 & 20 \\
\hline Sensitivity $(\mu \mathrm{m} / \mathrm{Pa})$ & 0.15 & $43.54 \mathrm{e}^{3}$ & $31.53 \mathrm{e}^{3}$ \\
\hline $\begin{array}{l}1^{\text {st }} \text { Resonant } \\
\text { Frequency }(\mathrm{Hz})\end{array}$ & - & 636.33 & 644.95 \\
\hline $\begin{array}{l}2^{\text {nd }} \text { Resonant } \\
\text { Frequency }(\mathrm{Hz})\end{array}$ & 1690 & 984.24 & 965.86 \\
\hline
\end{tabular}

\section{Conclusion}

MEMS based acoustic sensors have variety of applications. Reliability along with optimized output is significant for operation, which requires choosing of best fabrication process and its analysis. A MetalMUMPs based directional finding sound sensor was presented in this paper for particular application. The sensor output was compared with the output of sensor made from SOIMUMPs fabrication process. The sensitivity of the sensor has improved to $43.54 \mathrm{~mm} / \mathrm{Pa}$ when compared with the sensor of similar dimensions made from SOIMUMPs whose sensitivity is $0.15 \mu \mathrm{m} / \mathrm{Pa}$. It was also revealed that the sensitivity has increased when the strength of coupling was reduced. Stress analysis of the structure was carried out to estimate the operating range of the sensor. A technique of

modification in design is presented to reduce the stress concentration and further change in design is recommended to bring it in required operational frequency range.

\section{References}

[1] N. Islam and A. N. Nordin I. Voiculescu, Acoustic Wave Based MEMS Devices, Development and Applications in Microelectromechanical Systems and Devices., 2012.

[2] Nance D., Ahuja K. K. Kaushik B., "A Review of the Role of Acoustic Sensors in the Modern Battlefield," in 11th AIAA/CEAS Aeroacoustics Conference, Monterey, CA, USA, 23-25 May 2005.

[3] Maher, R.C., "Acoustical characterization of gunshots" IEEE Workshop on Signal Processing Applications for Public Security and Forensics, Washington D.C., 109-113, 2007.

[4] M. J. R. Lamothe and J. S. Bradley, "Acoustical characteristics of guns as impulse sources," in Can. Acoust. 13, 1985, pp. 16-24.

[5] Ylikoski M, Pekkarinen JO, Strack JP, Paakkinen RJ and Ylikoski JS. "Physical characteristics of gunfire impulse noise and its attenuation by hearing protectors" in Scand Audiol 24, 1995.

[6] Gun sound -- frequency analysis. [Online]. https://www.physicsforums.com/threads/gun-sound-frequencyanalysis.898882/

[7] Understanding Evolution.

[Online]. http://evolution.berkeley.edu/evolibrary/news/061201_quietcrickets

[8] W. Cade, "Acoustically orienting parasitoids: fly phontaxis to cricket song," in Science, vol. 190., 1975, pp. 1313-1313.

[9] Cornell Chronicle.

[Online]. http://www.news.cornell.edu/chronicle/01/4.19.01/fly-hearing.html.

[10] R.N. Miles, D. Robert, and R.R. Hoy. "Mechanically coupled ears for directional hearing in the parasitoid fly Ormia Ochracea." The Journal of the Acoustical Society of America vol. 98 pp. 3059-3070, December 1995.

[11] M. L. \& Hall, N. A. Kuntzman, "Sound source localization inspired by the ears of the Ormia ochracea," Appl. Phys. Lett. 105, 033701, 2014

[12] Luke Currano, Danny Gee, Benjamin Yang, and Miao Yu Haijun Liu, "Fly-ear inspired acoustic sensors for gunshot localization," Proc. of SPIE, vol. 7321,73210A-1, 2009.

[13] Murat Akcakaya and Arye Nehorai, "Performance analysis of the Ormia ochracea's coupled ears," Acoustical Society of America, pp. 2100-2105, 2008.

[14] Andrew Reid, Deepak Uttamchandani and James F.C. Windmill, "Bio-inspired sound localization sensor with high directional sensitivity," EUROSENSORS, 2015.

[15] M. Touse, J. Sinibaldi, K. Simsek, J. Catterlin, S. Harrison, and G. Karunasiri, "Fabrication of a microelectro mechanical directional sound sensor with electronic readout using comb fingers," Appl. Phys. Lett. 96, 173701, 2010.

[16] Rudresh. KJ, Veda Sandeep Nagaraja and Dr. SL Pinjare Nithya. G., "Design And Simulation of a Novel MEMS Acoustic Sensor," in COMSOL Conference in Bangalore, 2016.

[17] Fabio Alves \& Gamani Karunasiri Daniel Wilmott, "Bio-Inspired Miniature Direction Finding Acoustic Sensor," Nature, Scientific Reports, 2016.

[18] Allen Cowen, Bruce Dudley, Ed Hill, Mark Walters, Robert Wood, Stafford Johnson, Henry Wynands, Busbee Hardy: MetalMUMPs Design Book.

[19] He, Siyuan, et al. "Characterization of Young's modulus and residual stress gradient of MetalMUMPs electroplated nickel film." Sensors and Actuators A: Physical 154.1, pp. 149-156, 2009.

\section{About Authors :}

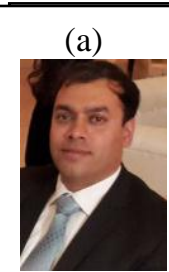

(c)

\section{(b)}

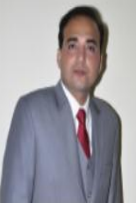

(d)

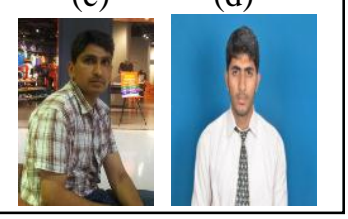

The authors are student Engineering Department, National University of Sciences and Technology, Pakistan. 